\title{
Evaluación de Competencias en Educación Superior: Experiencias en el Contexto Mexicano
}

\section{Competencies Assessment in Higher Education: Experiences in the Mexican Context}

\author{
Frida Díaz Barriga Arceo * \\ Universidad Nacional Autónoma de México, México
}

\begin{abstract}
Se ofrece un balance de la incursión del enfoque de competencias en la educación superior mexicana durante las últimas décadas con relación a distintas tendencias y procesos de apropiación. Con fundamento en la corriente sociocultural y el enfoque de evaluación auténtica, se discute el tema de la enseñanza y evaluación de competencias profesionales. Asimismo, se ofrecen dos ejemplos de buena práctica en el contex to mexicano: un modelo de portafolio electrónico de evidencias con docentes de educación básica en formación y un sistema de evaluación clínica objetiva estructurada (ECOE) en odontología. En ambos casos, se logró la comprensión y apropiación del enfoque en las comunidades educativas y se promovieron cambios en los procesos de gestión curricular que favorecieron su concreción. El artículo concluye recapitulando una serie de supuestos y condiciones asociadas a la instauración apropiada de sistemas de evaluación auténtica de competencias en el nivel superior.
\end{abstract}

Palabras clave: Competencias profesionales; Evaluación auténtica; Portafolio de evidencias; Competencias clínicas.

\begin{abstract}
A balance of the rapprochement of the competency approach in Mexican higher education during the last decades in relation to different tendencies and processes of appropriation is offered. Based on the sociocultural current and the authentic assessment approach, the teaching and evaluation of professional competences is discussed. Likewise, two examples of good practice in the Mexican context are offered: a model of electronic portfolio for teacher training and an objective structured clinical examination (OSCE) system in dentistry. In both cases, the understanding and appropriation of the approach in the educational communities was achieved and changes in the curricular management processes that favored its concretion were promoted. This paper concludes with a series of assumptions and conditions associated with the appropriate establishment of systems for authentic assessment of competences at higher level education.
\end{abstract}

Keywords: Professional competences; Authentic evaluation; Evidence portfolio; Clinical competences.

*Contacto: diazfrida@prodigy.net.mx

issn: 1989-0397

www.rinace.net/riee/

https://revistas.uam.es/riee
Recibido: $\quad 12$ de mayo de 2019

$1^{\text {a }}$ Evaluación: 11 de septiembre de 2019

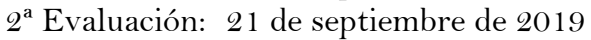

Aceptado: $\quad$ 24 de septiembre de 2019 


\section{Introducción}

A partir de la década de los noventa del siglo antecedente, el tema de las competencias, su enseñanza y evaluación, pasó a conformar parte del discurso hegemónico de la entrada a la sociedad del conocimiento y planteó como imperativo la necesidad de innovar estructuras y prácticas curriculares en la educación superior en tal dirección. Desde entonces, las reformas y modelos curriculares se han caracterizado en nuestro contexto, así como en muchos otros países (no sin una buena dosis de controversia) por arquitecturas curriculares y programas basados en la delimitación de competencias tanto de orden genérico o transversal, como específicas de diversas profesiones. Esta tendencia se ha visto acompañada por un replanteamiento de la evaluación de los profesionales, ajustada a la demostración de desempeños ajustados a estándares profesionales, ya sean locales o internacionales.

Un claro ejemplo de lo anterior, con trascendencia en el contexto iberoamericano, es el modelo Tuning Europa, que en su momento derivó en Tuning América Latina, en el cual 62 instituciones de educación superior (IES) públicas y privadas de 18 países de la región identificaron las competencias genéricas y específicas propias de diferentes áreas de conocimiento, con la intención última de poder comparar y reconocer las titulaciones entre dichos países, lo que beneficiaría la movilidad de los profesionales y la calidad de la formación (González, Wagenaar y Beneitone, 2004). Con independencia del impacto del proyecto, es de reconocer que emerge del reconocimiento de sendos procesos de internacionalización de las profesiones, en lo que atañe a la formación a nivel superior, la certificación de los profesionales y la acreditación de los currículos e instituciones formadoras.

El interés por el currículo y la evaluación de competencias no sólo proviene del sector educativo, sino de manera destacada, del económico y laboral. Al respecto, Barrón (1997) afirma que a finales del siglo veinte ya era más que evidente el peso de las políticas globales en materia educativa, sobre todo en la formación de los profesionales (técnicos y universitarios) como respuesta a un nuevo orden mundial y debido a las presiones provenientes de los organismos internacionales que impulsaron políticas de educación basada en competencias, la apropiación de innovaciones tecnológicas asociada a determinadas profesiones, así como la posibilidad de conformar trayectos formativos flexibles y adaptables a la vorágine de cambios continuos en el sector laboral.

En 2003 W. Pinar edita el International Handbook of Curriculum Studies, que constituye el primer intento por configurar la arquitectura de los estudios curriculares a lo largo del mundo a través de discutir la historia y el estado actual de este campo de investigación e intervención en 29 países. Documenta la emergencia de importantes tensiones entre las perspectivas global y local. Para entonces, en varios países desde hacía por lo menos una década se hacían intentos por adoptar en las reformas curriculares nacionales los enfoques educativos basados en competencias, así como la evaluación por desempeños y proliferaban miradas propias de la visión neoliberal y empresarial de la educación. De forma destacada, la hegemonía de las evaluaciones estandarizadas y los enfoques de rendición de cuentas, aseguramiento de la calidad y reingeniería educativa tomaban un papel central en el control de los sistemas educativos.

En el estado de conocimiento sobre la investigación curricular que da cuenta de las reformas educativas de principios del siglo veintiuno en México, conducido por el Consejo 
Mexicano de Investigación Educativa (COMIE) se postula que prolifera el discurso de la innovación mediante la incorporación de tecnologías digitales, formación por competencias, currículo flexible y apertura a enfoques educativos experienciales y situados en escenarios profesionales (Díaz Barriga y Barrón, 2014). Se intenta cambiar la enseñanza tipo cátedra magistral y se exige al profesorado desarrollar programas por competencias, así como dar concreción en las aulas a una diversidad de innovaciones educativas. Los modelos curriculares incorporan la educación basada en competencias tanto en la educación básica obligatoria como en las instituciones de educación superior (IES), aunque cada cual entiende a su modo el concepto de competencia, su diseño y evaluación.

No tarda un surgir una importante corriente crítica de esta perspectiva y de la forma en que se introdujeron los enfoques curriculares y educativos basados en competencias en los currículos nacionales y de diversos países latinoamericanos: carentes de un marco conceptual propiamente educativo, con prescripciones técnicas confusas, sobre todo, con una evidente ausencia de buenos programas de formación de docentes o sin los debidos apoyos institucionales. En particular, los docentes de los campos de humanidades, arte o literatura se erigieron como los más críticos del enfoque, cuestionando el tipo de ser humano que se pretendía formar, diciendo que su inclinación era estandarizante y se quería transformar a la educación en "una fábrica de recursos humanos" al servicio de las empresas (Del Rey, 2012, p. 17). Autores como Ángel Díaz Barriga (2006) advirtieron que se estaba incursionando en el enfoque de competencias mediante propuestas poco sustentadas teórica y empíricamente, que conflictuaban la práctica docente y los fines de la educación, dado su énfasis tecnocrático. Asimismo, este autor cuestionó la introducción de supuestos expertos que comerciaban con "metodologías" carentes de lógica y rigor, por lo cual, en muchos casos, el supuesto plan de estudios por competencias se convirtió en un "disfraz de cambio".

A nuestro juicio, en México se llegó a enfrentar una situación similar a la reportada por Mulder, Weigel y Collins (2007) quienes analizaron el uso del término competencia en la formación profesional técnica en Inglaterra, Francia, Alemania y los Países Bajos. Encontraron que existían importantes dificultades para establecer una definición coherente y de uso común del concepto, que no era sencillo desarrollar estándares apropiados para el diseño y la evaluación de programas. Al parecer, entre los educadores se generó la concepción errónea de que la competencia va en detrimento del valor del conocimiento o que se pueden enseñar competencias desprovistas de contenido.

Otros estudios que resultan de interés son los que reporta Cano (2015) sobre la valoración que hacen más de 36000 egresados de 12 países europeos, en donde se concluye que las competencias transversales (resolución de problemas, adaptabilidad, toma de decisiones, trabajo en equipo, comunicación, etc.) son mucho más valoradas que las específicas de la profesión. Esto se corrobora según expone la autora, con otros estudios tipo encuesta realizados en España, además de que se encuentra que los titulados dicen sufrir un "déficit competencial" cuando salen de la universidad. Opiniones similares se encuentran entre el profesorado y los empleadores. En nuestro país no encontramos encuestas de tal alcance muestral, pero diversos estudios de caso en contextos locales dan cuenta de la complejidad del enfoque de competencias, de los cambios que implica, sobre todo por las tensiones que genera la evaluación y la carencia de acompañamiento al docente durante su implantación (López-Figueroa, 2014). 
En coincidencia con los estudios de los sociólogos del cambio educativo, cuando se emprenden ambiciosas reformas nacionales, como es el caso de la instauración de modelos como el de competencias, es menester un abordaje sistémico (Fullan, 2001). Esto no es de extrañar, porque la verdadera innovación requiere cambio de mentalidades y prácticas socioculturales. Es decir, se requieren procesos de apropiación y cambios de fondo en las comunidades educativas: formación y condiciones propicias para los profesores, replanteamiento de la organización y gestión institucional, promoción de trayectorias de aprendizaje flexibles y personalizadas, por ende, cambios en la programación educativa. Ante todo, habrá que lograr la concreción en las aulas y espacios educativos, no sólo en los documentos base del currículo.

Cuando se pretenden innovaciones de gran envergadura, se enfrenta la transformación del rol e identidad del profesor y del estudiantado, e incluso del personal directivo y otros encargados de la gestión. Sin embargo, un aspecto que no parece apoyar la diseminación apropiada ni la aceptación entre los actores del currículo se relaciona con la rigidez de los diseños curriculares, la estandarización y los procesos de evaluación que siguen anclados en la reproducción de contenidos. El potencial de un modelo curricular por competencias reside en una perspectiva abierta, flexible y mutable ante las posibilidades del contexto donde se desarrolle el conocimiento en la acción. Sin embargo, en paralelo a la instauración del enfoque de competencias, en nuestro país predominaron las políticas que impulsaron la evaluación estandarizada de largo alcance controlada por organismos nacionales e internacionales, con instrumentos que fueron ampliamente cuestionados tanto por sus usos como por el tipo de aprendizajes (reproductivos, declarativos, descontextualizados) que evalúan.

En el contexto internacional, Hargreaves (entrevistado por Romero, 2008, p. 65) encuentra que las reformas curriculares por lo general y en franca contradicción con la innovación que enarbolan, "han optado por la estandarización en detrimento de la creatividad, la flexibilidad, el sentido de comunidad y la escuela democrática e inclusiva”.

Consideramos que el enfoque por competencias (como cualquier otra innovación curricular), cuando pretende instaurarse como prescripción técnica, sin una reflexión conceptual y un marco teórico de referencia, suele caer en una visión pragmática y reduccionista. Esto conduce a que el diseño y evaluación de competencias, quede reducido al dominio de un "saber hacer" procedimental puntual y fuera de contexto, como una vía que sólo permite definir registros de tareas o comportamientos discretos y fragmentados. En particular cuando se intenta pasar de la lógica de las competencias técnico-laborales a la definición de competencias académicas, investigativas y profesionales, este tipo de enfoque de entrenamiento de tareas discretas resulta inapropiado e inoperante.

Ahora bien, la visión empresarial de las competencias no fue la única con una presencia importante en el país. Se desarrollaron otras perspectivas que intentaban dotar de un sentido pedagógico y social al tema. En particular, las perspectivas constructivista y sociocultural, así como los enfoques de enseñanza situada y solución de problemas pretendieron llenar el vacío teórico y pedagógico del abordaje de las competencias en la educación. En el plano académico destaca la influencia de la concepción de competencia propuesta por autores de la corriente francófona como Jonnaert (2002), Perrenoud (2004), Denyer, Furnémont, Poulain y Vanloubbeeck (2007), entre otros, caracterizada como socioconstructivista, que aporta otra lógica de transposición didáctica y apela al desarrollo de situaciones didácticas y para la evaluación. En este caso, la competencia implica 
movilizar e integrar diversos saberes y recursos, poder re-co-construir el conocimiento en una situación y contexto específico, proponer una solución o tomar decisiones pertinentes. Implica al mismo tiempo una acción autorregulada, consciente, realizada con conocimiento de causa, que involucra saber, saber hacer y ser. El punto de partida lo constituyen situaciones problema de relevancia social, en torno a las cuales se construyen situaciones de aprendizaje constructivo, complejo y colaborativo, para la solución de problemas de índole interdisciplinar preferentemente, que se desarrollan en escenarios reales o mediante simulaciones situadas. Se pone en relieve el compromiso social de la formación, más allá de los intereses de la empresa.

$\mathrm{Al}$ respecto, César Coll (2007) afirma que la noción sociocultural de las competencias hace contribuciones importantes a la educación, puesto que ubica en un primer plano la importancia de la funcionalidad y significatividad de los aprendizajes logrados. También introduce la idea de que es indispensable arribar a la integración y dinamización de distintos tipos de saberes, a la par que reconoce la especificidad de lo que se enseña y evalúa, su carácter situado. Para este autor, las competencias son "capacidades situadas" y son relevantes en una sociedad donde ha cambiado la ecología del aprendizaje, ante las demandas actuales de la sociedad y la necesidad de formar personas a lo largo y ancho de la vida.

¿Hacia dónde van las competencias hoy en día? Algunos autores (Loveless y Williamson, 2017) consideran que dicho enfoque seguirá vigente, pero desde una perspectiva distinta, en la que, por lo menos en las sociedades avanzadas y crecientemente tecnificadas, el aprendizaje se distribuirá en ecosistemas de aprendizaje en red, entre escenarios virtuales y reales de tipo formal e informal. Ante esto, la concepción misma de asignaturas y de currículo, como la entendemos, desaparecerá en su momento. Los aprendientes tendrán que generar nuevas identidades de aprendiz, en las que no sólo consumirán información o contenidos curriculares estáticos sino, ante todo, necesitarán competencias para gestionar la información, para lograr una comunicación y colaboración efectivas, para solucionar problemas, así como para producir conocimiento, publicarlo y compartirlo. Las escuelas y universidades serán una especie de "nodos" dentro de una red de espacios colaborativos de aprendizaje. Se habla de una educación orientada al desarrollo de literacidades (digital, ciudadana, académica, etc.) que se definen en término de competencias o capacidades situadas, complejas, dinámicas e incrementales, que permiten a la persona conformar su propia trayectoria de aprendizaje o auto-currículo.

No existen perspectivas "asépticas" en la evaluación, ya que toda evaluación cubre una función social que es dependiente de una serie de intereses, contextos y valores culturales, no sólo académicos. Por ende, el asunto de la enseñanza y evaluación por competencias no puede sustraerse al escrutinio de sus fines y usos. Consideramos que se necesita contrastar dicha mirada con la situación de diversidad y desigualdad que enfrentan las instituciones educativas de nuestro país, donde no dudamos que pueda haber contextos propicios para la mejor expresión de dicha prospectiva, pero seguramente habrá también contextos donde esto resulte inviable o donde genere más desigualdad y resistencia entre los actores del currículo. A nuestro juicio en nuestro contexto se enfrenta una importante tensión: los modelos curriculares prescriben competencias, mientras que las políticas educativas de largo alcance están centradas en exámenes escritos estandarizados.

Tal vez la reflexión más trascendente sobre el tema de la evaluación y sus contradicciones, dado el peso que se le da a la evaluación mediante exámenes para rendir cuentas desde una 
visión administrativa, es que ésta se ha convertido en el eje rector que configura la enseñanza y el aprendizaje. Según Stobart (2010) la evaluación no mide objetivamente lo que hay, sino que crea y configura lo que mide, por eso puede afirmarse que la filosofía y práctica de la evaluación están teniendo un peso muy importante en la construcción de la identidad de los aprendices. Para el autor, debe ser motivo de preocupación si lo que se transmite a los futuros profesionales es que la meta es trabajar más que nada por las calificaciones y los títulos.

\section{Adquisición y evaluación de competencias profesionales}

Sabemos que las competencias profesionales se desarrollan mediante procesos de formación deliberados y dirigidos, aunque también se desarrollan y perfeccionan en el devenir cotidiano del practicante cuando enfrenta las situaciones clave de la práctica en escenarios reales. De acuerdo con el modelo del profesional reflexivo de Donald Schön (1992) lo que marca la diferencia en el desempeño de los profesionales es su capacidad de contender con las situaciones que son propias de un contexto profesional en cambio permanente. El autor las denominada "zonas indeterminadas de la práctica", caracterizadas por la incertidumbre, la singularidad y la presencia de conflictos de valor. Los problemas relevantes que se enfrentan en el mundo profesional, y en general en los escenarios de la vida real, son problemas abiertos; constituyen situaciones poco definidas o estructuradas, y plantean al profesional dilemas de diversa índole (de desarrollo tecnológico, ambientales, éticos, políticos, económicos). Un profesional competente será aquél que sepa manejar de manera conveniente dichas situaciones inéditas pero pertinentes a su campo de acción profesional; requiere tomar iniciativas y decisiones, negociar, hacer elecciones en condiciones de riesgo, innovar y asumir responsabilidades.

Por ende, para enseñar-aprender-evaluar competencias, se requiere crear situaciones didácticas explícitas y pertinentes, que permitan enfrentar directamente a los aprendientes a las tareas complejas (generativas, orientadas a la solución de problemas) que se espera resuelvan. Se requiere que adquieran y aprendan a movilizar los recursos indispensables y que lo hagan con fundamento en procesos de reflexión metacognitiva o autorregulación. En buena medida, la clave de la enseñanza y evaluación de competencias es la pertinencia que guardan con los contextos socioculturales de adquisición y uso de tales competencias.

La metáfora experto-novato suele ser un modelo empleado en la enseñanza y la evaluación de competencias profesionales. Parte del supuesto que existe un camino o trayecto a recorrer en la profesión, en el transcurso del cual la persona en formación se enfrentará gradualmente al ejercicio de la práctica en escenarios reales, lo que le permitirá desarrollar la pericia esperada, adquirir una serie de destrezas y perfeccionar su desempeño. Lo que diferencia al experto del novato consiste en la forma en que el primero organiza y estructura el conocimiento, la profundidad y complejidad con la que puede representarse y afrontar una situación-problema y las habilidades metacognitivas (de comprensión y regulación de la propia acción) que despliega. Conforme los profesionales en proceso de formación ganan en experiencia y conocimiento, cambia la representación que tienen de su ámbito de intervención, de su propio rol y de las personas a quienes destinan su labor, al mismo tiempo que aprenden a codificar reglas y principios basados en su conocimiento práctico (qué hacer y cómo hacerlo) (Schön, 1992). 
Por otra parte, las competencias se aprenden y se validan en el seno de una comunidad de práctica (Wenger, 2001). La manera en que los profesionales perciben y emplean determinadas herramientas físicas o simbólicas, en que definen e intervienen en las situaciones problema e incluso en la forma en que organizan el conocimiento, depende no sólo de sus historias de vida personales, sino de los saberes aprendidos en el seno de la comunidad de práctica profesional a la que pertenecen o aspiran pertenecer. Una comunidad de práctica comparte creencias y enfoques hacia lo que implica aprender y enseñar, así como prácticas, herramientas y tecnologías, valores, lenguajes, expectativas y metas. Las universidades en las que se forman los profesionales vehiculan estas miradas de las comunidades profesionales, imprimiéndoles su sello característico. las decisiones respecto al qué y al cómo del currículo son una de sus expresiones más importantes de la presencia de una determinada comunidad de práctica profesional. Por ejemplo, no es lo mismo aprender psicología en un programa orientado hacia el experimentalismo y el conductismo en una universidad pública, que formarse en la psicología humanista en el seno de una universidad privada. Las relaciones de participación, así como las identidades de los integrantes de la comunidad se transforman en función de las posibilidades y restricciones de los contextos donde se desenvuelven.

Si bien no existen situaciones didácticas únicas o idóneas que se puedan prescribir a priori para enseñar y evaluar competencias, el principio rector es que hay que enfrentar a la persona en formación a situaciones donde se recrean con la mayor autenticidad posible los problemas o casos propios de la práctica de la profesión. Por lo anterior es que los métodos educativos de indagación, experienciales y de formación en la práctica (aprendizaje mediante proyectos, aprendizaje basado en problemas, método de casos, aprendizaje servicio en la comunidad, entre otros) constituyen opciones viables (Díaz Barriga, 2006). La cuestión es que se requieren contextos de adquisición, uso y evaluación donde los profesionales en formación puedan afrontar tareas-problema reales y relevantes. Asimismo, Perrenoud (2004) considera que todo referencial de competencias "pasa de moda" porque las profesiones no son inmutables, sus prácticas cambian y el modo de concebirlas se transforma.

Roe (2003) afirma que no existen profesionales generales, porque el ejercicio profesional implica una cierta especialización, muy ligada al contexto y a la dinámica particular de la profesión en un país determinado. Considera asimismo que no todo lo que se enseña en las universidades puede categorizarse como competencia y que las competencias profesionales están sujetas a un proceso formativo complejo, gradual y temporal. Para que se adquieran competencias es condición trabajar con los estudiantes con un enfoque de problemas que los lleve a integrar y movilizar el conocimiento adquirido. Lo interesante también es entender que la formación de competencias profesionales puede o no ocurrir durante los estudios universitarios, ello depende de las experiencias de formación en que participen los alumnos. Es decir, un estudiante puede tener determinados conocimientos y capacidades, pero no ser capaz de integrarlos o emplearlos frente a un problema real que tiene que enfrentar; de ahí que poseer conocimiento de teorías y métodos no es garantía de un desempeño competente en la profesión. Los planteamientos antecedentes son importantes porque enmarcan el sentido y la complejidad del tema de la evaluación de competencias.

Para evaluar la posesión de una competencia es necesario demostrar que se cubren a satisfacción las exigencias de la tarea, que se saben realizar las funciones que requiere y se manifiesta el tipo y nivel de desempeño esperado, el cual resulta satisfactorio, eficaz o 
exitoso en cuanto lo que aporta o soluciona. De acuerdo con Cano (2015), implica un proceso en el que se recoge información acerca de la evolución y las ejecuciones de los estudiantes, se valora a la luz de unos criterios que conducen a tomar decisiones. Se requiere claridad respecto al objeto de la evaluación y el tipo de contenido que se pretende evaluar, logrando conclusiones válidas, fidedignas, libres de sesgos prejuiciados.

La evaluación de competencias se centra en el desempeño, que demanda a los estudiantes demostrar que poseen ciertas habilidades en situaciones de prueba ex profeso. A su vez, la evaluación auténtica (authentic assessment) va un paso más allá en el sentido que destaca la importancia de la aplicación de la habilidad en el contexto de una situación de la vida real propia del campo o profesión que se está valorando. La evaluación auténtica de competencias implica entender cómo ocurre el desempeño en un contexto y situación determinados, o seguir el proceso de adquisición y perfeccionamiento de determinados saberes o formas de actuación. Implica la autorregulación y autoevaluación de la persona en formación, puesto que destaca la construcción activa, propositiva y colaborativa del propio conocimiento (Darling-Hammond, Ancess y Falk, 1995).

El término auténtico o autenticidad se aplica en el caso de las competencias profesionales con relación a un contexto o dominio particular, a una comunidad de práctica que privilegia determinados saberes y prácticas sociales. Al construir una situación de evaluación, la autenticidad también abarca la posibilidad de una recreación lo más fiel posible del campo de transferencia del conocimiento y de sus condiciones habituales (tiempo, restricciones, acceso, lenguajes e instrumentos). Por ello se busca la evaluación de competencias en situaciones y casos reales, mediante proyectos o eventualmente a través de simulaciones situadas o casos realistas.

Existe una diversidad de estrategias e instrumentos congruentes con la perspectiva de la evaluación auténtica centrada en el desempeño: los portafolios, las pautas de observación y/o autoevaluación de una ejecución, las pruebas situacionales, los registros observacionales y anecdóticos, los diarios de clase, las bitácoras o blogs, las rúbricas o matrices de valoración, entre otros. Sin embargo, el uso de estos instrumentos, por sí mismo, no es garantía de la adopción de la evaluación auténtica del desempeño.

Por otra parte, una exigencia que no siempre se cumple, consiste en la congruencia entre lo que se enseña y lo que se evalúa. Se propone que la situación en la cual se enseña y aprende, es en sí misma la que ofrece la oportunidad de una evaluación formativa y comprensiva. Es decir, la evaluación en sí misma es una oportunidad de aprender mediante la retroalimentación, la autoevaluación, la práctica reflexiva y correctiva realizando un análisis sistémico del acto educativo.

También hay que decir que la evaluación auténtica privilegia el abordaje cualitativo sobre el cuantitativo (sin excluirlo si se considera pertinente) y propugna por el empleo de múltiples instrumentos y fuentes de información o piezas de evidencia para determinar si se ha logrado o no el aprendizaje de un saber o competencia y en qué nivel se ubica el mismo.

Lo importante es proveer elementos para la comprensión, retroalimentación y mejora del aprendizaje y del proceso educativo. De acuerdo con Cano (2015) es importante transitar de una retroalimentación o feedback tradicional centrado en el criterio del docente, a un feedback dialógico e interactivo, pertinente a desempeños futuros. 


\section{Dos experiencias de evaluación auténtica de competencias en el nivel superior}

En esta sección se expondrán dos experiencias de evaluación auténtica de competencias para la profesión en el contexto de la educación superior mexicana. No es la pretensión plantear que constituyen modelos paradigmáticos ni generalizados o incluso pertinentes a todas las IES, dado su carácter situado. Sin embargo, constituyen ejemplos exitosos de prácticas alternativas de evaluación de competencias que han logrado el compromiso y la participación de las comunidades educativas de referencia. Más que nada, han coadyuvado a una visión de enseñanza-evaluación alternativa y auténtica en el sentido que aquí se ha venido exponiendo.

\subsection{Caso 1. Portafolios de evidencia de estudiantes normalistas}

Los portafolios de evidencia han sido ampliamente impulsados en sendos programas de formación y evaluación del profesorado, sobre todo en los contextos anglosajón, canadiense y europeo, mientras que en la región latinoamericana destacan distintas experiencias chilenas y mexicanas. En algunos casos, se han logrado documentar experiencias de buena práctica en cuanto al diseño e instrumentación del portafolio docente como recurso de evaluación formativa y reflexión en profesores nóveles. Permiten organizar, dar a conocer y evaluar el desempeño de los docentes, así como para promover su capacidad profesional y reflexión personal. El creciente interés por su empleo a partir de la década de los ochenta se relaciona con la formación y evaluación por competencias.

En la experiencia que se relata (Díaz Barriga y Barroso, 2015), se aborda la modalidad de portafolio electrónico (e-portafolio) y se presentan los componentes clave de un modelo desarrollado con jóvenes de escuelas normales mexicanas públicas, que se encontraban a punto de concluir sus estudios e iniciar su trabajo profesional. La realización del portafolio electrónico durante el último año de estudios (séptimo y octavo semestre de la Licenciatura en Educación Preescolar en la Escuela Normal de Educación Preescolar de Oaxaca, México) les permitía a los futuros docentes graduarse de dicha licenciatura. Cabe aclarar que en México las escuelas normales son las responsables de la formación del profesorado de educación básica.

Se definió al portafolio docente como una selección o colección de trabajos o producciones elaborados por el profesor en formación como evidencia de la planeación, conducción y evaluación del proceso enseñanza-aprendizaje de sus alumnos. Dichos trabajos han sido realizados en el transcurso de un ciclo de prácticas en un escenario preescolar con base en un proyecto didáctico propio. El portafolio fue concebido como dispositivo pedagógico de formación, evaluación y autorregulación del aprendizaje de las competencias clave de un docente de preescolar. Se construyó en formato electrónico para promover la literacidad digital de los normalistas, para que lograran un uso didáctico de recursos multimedia (sonido, gráficos, video, hipertexto) y de herramientas sociales de internet. Se vincularon una diversidad de rúbricas de desempeño para la auto-co-hetero evaluación del portafolio y de las competencias docentes, así como un diario interactivo para que el asesor de los normalistas pudiera retroalimentar de manera dialógica sus proyectos didácticos y desempeños en el escenario preescolar. También se planteó la incursión de un par o compañero de aprendizaje, integrante del grupo de estudiantes, para propiciar el intercambio de experiencias y materiales en una dinámica de aprendizaje mutuo y 
colaboración. Como componente de investigación, el énfasis se ubicó en identificar los procesos inherentes al pensamiento reflexivo y la autoevaluación.

El proyecto incluyó dos metodologías: la investigación basada en el diseño y el estudio de casos. La investigación basada en diseño (DBR), que consiste en "estudios de campo, en los que un equipo de investigación interviene en un contexto de aprendizaje particular para atender, mediante un diseño instructivo, al logro de una meta pedagógica explícitamente definida” (Design-Based Research Collective, 2003, p. 5). Es decir, pretende entender las relaciones entre las teorías o modelos educativos, los artefactos y actividades instruccionales en la realidad cotidiana de la práctica educativa en distintos contextos. Su contribución reside en la elaboración de marcos explicativos con fundamento empírico y situados en contextos educativos naturalistas o auténticos. Por lo general, se procede en subsecuentes ciclos de diseño, para poder ajustar el modelo y las ayudas pedagógicas en diversos episodios didácticos.

En el primer ciclo, participaron 36 estudiantes de la licenciatura referida, 34 mujeres y 2 varones, con una moda de 22 años, y cuyas actividades de práctica se realizan en distintos lugares del estado de Oaxaca, por lo que destaca la diversidad socioeconómica, cultural e incluso étnica de los escenarios educativos. Algunos jóvenes se ubicaban en preescolares de la periferia de la capital del estado mientras que otros estaban en escuelas con población indígena en zonas semi-rurales.

En un inicio y en todos los casos, los normalistas procedieron con el acompañamiento de su asesor, a realizar un estudio de caso de tipo etnográfico y participativo del escenario preescolar donde realizaban sus prácticas, lo que condujo a problematizar las características, necesidades e intereses de la comunidad educativa. A partir de ello, se configuró un diseño educativo (situación didáctica) para el escenario en cuestión y la ruta crítica de intervención. El portafolio electrónico de evidencias se basó en las metáforas de espejo (identidad docente), mapa (evidencias y reflexiones) y soneto (expresión creativa y personalización de trayecto docente) propuesta por Gibson y Barrett (2003). Las entradas o secciones del portafolio incluían:

- Bienvenida y sentido del portafolio

- Acerca de mí (narrativa autobiográfica)

- Escuela y contexto

- Planeación para el aprendizaje

- Organización del ambiente en el aula

- Evaluación educativa

- Promoción del aprendizaje de todos los alumnos (Aula Inclusiva)

- Identidad profesional y ética

- Vinculación con la institución y el entorno

- Mi documento recepcional

- Visión prospectiva del plan de vida y carrera

Se delimitaron competencias generales y específicas vinculadas con el currículo y se plantearon propósitos de evaluación, evidencias de aprendizaje, criterios de desempeño y preguntas de reflexión. Se consideraron la hetero, co y auto evaluación, así como diversos 
instrumentos en función de su pertinencia y momento del proceso educativo: rúbricas, diario interactivo, observaciones, narrativa autobiográfica, entre otros.

Para validar el modelo de portafolio, se integró una muestra de 132 docentes de diversas escuelas normales mexicanas, todos ellos profesores titulares, quienes respondieron un instrumento tipo Likert autoadministrado en línea. Se encontró que nueve de cada diez docentes estaban de acuerdo o completamente de acuerdo, en que el sistema de evaluación mediante el portafolio y el proceso que conduce a la generación del documento recepcional, permiten guiar el aprendizaje de los estudiantes y coadyuvan en las decisiones que el docente debe tomar para la mejora de la enseñanza, lo que indica que es la dimensión formativa la más destacable en este dispositivo. Consideran que los estudiantes avanzan en desarrollar una mirada autocrítica y a la vez propositiva de su actuación y producciones, además de que se evidencian tanto los procesos como las situaciones más relevantes en que se ve involucrado el futuro docente. Ulteriormente, se han conducido otros ciclos de diseño para perfeccionar la propuesta y desarrollarla en el contexto específico de otras escuelas normales en diversos estados del país.

El avance más reciente de este proyecto consiste en una app denominada Aplicación Móvil del Diario Interactivo (AMDI) que se puede descargar gratis al teléfono celular desde Google Play Store y funciona para sistema operativo Android. En esta versión de diario de aprendizaje interactivo, se dispone de opciones para personalizar el diario, generar narrativas identitarias, compartir experiencias de aprendizaje, crear grupos de colaboración e intercambio, interactuar con el asesor y compañero de aprendizaje elegido, vincularse a redes sociales (Facebook), navegar en la red (a través de Google), incluir video, fotografía, notas de voz y audio en lo que se publica. También se puede cargar documentos en distinto formato (Word, PDF, Excel). La meta es crear un ambiente virtual de interactividad orientado a promover y evaluar los procesos reflexivos vinculados al aprendizaje de las competencias del futuro docente (Barroso, 2017; Barroso y Siqueiros, 2015). Los resultados de esta experiencia, en sus distintos ciclos de trabajo, es que lo profesores logran una mayor conciencia y reflexión acerca de las competencias que tienen que desplegar en el escenario educativo donde concluyen su formación profesional, avanzan en el manejo de dispositivos digitales y didácticos en la enseñanza, y ante todo, en la posibilidad de comprender las necesidades de la comunidad educativa y de los educandos de preescolar, por lo que las secuencias didácticas que proponen, realmente están centradas en el sujeto de la educación. Otro resultado de interés reside en la importancia del tipo y cualidad de la acción tutorial que requiere un docente novato que inicia su trayecto profesional.

\subsection{Caso 2. Sistema ECOE para la evaluación de competencias de odontólogos en formación}

Otro sistema de evaluación de competencias que vale la pena resaltar en el campo de la educación médica y de la salud es la evaluación clínica objetiva estructurada (ECOE). Este sistema fue introducido en el campo de la medicina a partir de 1975 por Harden y colaboradores (en Espinosa, 2018), como un sistema de evaluación en que los médicos en formación o ejercicio rotan en un circuito de "estaciones de competencia” en las cuales se desempeñan en una tarea clínica para demostrar sus capacidades en un dominio específico de la profesión. Se le llama evaluación estandarizada con la idea de que todos los evaluados tengan condiciones similares de valoración de la competencia, en cuanto al tipo de objetivo, lugar donde se desarrolla, material requerido, instrucciones, tipo de caso clínico, 
tiempo, complejidad de la tarea, etcétera. Resulta esencial disponer de un conjunto acotado y validado por expertos en la materia- de escalas de desempeño, usualmente rúbricas, pautas de interrogación y hojas de respuesta. Los evaluadores, expertos del campo competencial, deben estar entrenados previamente y mostrar un alto índice de confiabilidad en la evaluación del desempeño en cuestión. Las estaciones de competencia pueden clasificarse en dos tipos: a) estaciones dinámicas, donde el practicante desempeña competencias propias de una tarea clínica con un paciente estandarizado (PE) o en un simulador de desempeño; y b) estaciones estáticas, en las que tiene que responder cuestionamientos sobre la base de la información que ha obtenido en la misma estación. El paciente estandarizado es un adulto o niño que ha sido entrenado para recrear de forma realista y precisa la historia médica, síntomas físicos y respuestas emocionales típicas de un paciente real en el caso a tratar (Espinosa, Martínez, Sánchez y Leenen, 2017).

Este sistema ha sido probado en la carrera de medicina y recientemente en odontología. En este último caso, el propósito del modelo ha sido evaluar el desarrollo de los dominios de la competencia clínica en estudiantes de pregrado en lo que atañe a odontopediatría, que se imparte en el cuarto año de la licenciatura en Cirujano Dentista en la Facultad de Odontología de la Universidad Nacional Autónoma de México (UNAM). El proceso de evaluación implica trabajo de investigación y un seguimiento cuidadoso, con la intención de aportar evidencia científica y perfeccionar el sistema. Se realizó un estudio longitudinal pre-postest, es decir, se aplicó el sistema ECOE antes y después del curso de Odontopediatría. Consistió en un circuito de 18 estaciones con duración de 6 minutos cada una. También se realizó un estudio cualitativo por medio de grupos focales para conocer la opinión de los estudiantes sobre el ECOE ${ }^{1}$.

En el primer ciclo, participaron 120 estudiantes y se encontró un progreso significativo, corroborado estadísticamente, en el desarrollo de la competencia clínica en odontopediatría, principalmente en los dominios de plan de tratamiento y habilidades técnicas. Se pudieron identificar de manera consistente y en profundidad las habilidades que son desarrolladas adecuadamente y las que no durante el ciclo escolar, lo que aporta elementos para la retroalimentación tanto de la institución formadora como de docentes y alumnos.

También se encontró que los aspectos que confirieron más validez al sistema ECOE empleado fueron la creación de una apropiada matriz de competencias, la representatividad de las estaciones creadas, la inclusión de casos situados y reales, el uso de distintos recursos materiales y tecnológicos para estructurar las estaciones, acorde a los escenarios de formación-evaluación de esta profesión. Dado el tipo de información arrojada, se pudieron analizar los resultados de diversos dominios de competencia (exploración física, diagnóstico e interpretación radiográfica, entrevista, prevención y promoción de la salud, etcétera) y recuperar la perspectiva de los estudiantes respecto a cada uno de éstos.

Entre los factores que contribuyeron a la adecuada operación del ECOE se encuentran la adecuada capacitación y manejo de los pacientes estandarizados, que en este caso fueron niños y niñas quienes, desde la perspectiva de los estudiantes evaluados, permitieron que las estaciones se percibieran como reales. Su actuación permitió explorar adecuadamente

\footnotetext{
${ }^{1}$ Véase ECOE en Odontología UNAM en https://www.youtube.com/watch?v=SV1X7CdoCxU
} 
habilidades de comunicación, profesionalismo o exploración física. También resultó muy relevante el papel de los examinadores en la operatividad del sistema, y se reiteró que es muy importante capacitarlos.

Las rúbricas fueron de particular utilidad para estandarizar los criterios de respuesta de los profesores evaluadores. Con las rúbricas diseñadas (se fueron ajustando conforme sus resultados) se pudo hacer una distinción clara de las opciones de respuesta en cuatro niveles; la descripción de cada nivel de criterio fue mutuamente excluyente y no permitió que pudiera ser confundida o traslapada con la opción de respuesta de otro nivel del mismo criterio (Espinosa, 2018).

En síntesis, esta experiencia de evaluación auténtica permitió valorar el aprendizaje de la competencia clínica en odontopediatría y resultó apropiada para el saber hacer técnicoprocedimental, así como para identificar la coherencia entre la didáctica y la evaluación.

Resulta de interés destacar la importancia de que los docentes, expertos en el campo de la profesión, estén formados en el tema de la enseñanza y evaluación de competencias, sean entrenados hasta lograr evaluaciones consistentes y con el suficiente nivel de acuerdo con otros evaluadores, comprendan el cómo y para qué se emplean determinados instrumentos, como las rúbricas de evaluación del desempeño, y logren alinear el contenido curricular con las evidencias de desempeño que se muestran al valorar las competencias.

\section{Conclusiones}

En este escrito hemos argumentado que no existe una sola forma de concebir el diseño y la evaluación de competencias en la educación, y que la operación de dicho enfoque en las instituciones con frecuencia se ve obstaculizada por una diversidad de factores. La dispersión de concepciones, la falta de claridad teórica y de capacitación del profesorado, la carencia de un abordaje sistémico y condiciones para un cambio educativo que modifique el paradigma reproductivo-expositivo, son algunos de los más relevantes. No obstante, el enfoque de competencias y la perspectiva de una evaluación auténtica de las mismas es de interés en la medida en que se adopte una perspectiva que vaya más allá de una prescripción técnica y una visión restringida a calificar conductas discretas o productos fuera de contexto.

La potencialidad del enfoque reside en una concepción de competencia como prescripción abierta, que da la pauta a entender y promover aprendizajes situados, focalizados en la construcción e innovación del conocimiento, el afrontamiento de situaciones problema de relevancia personal, social y profesional. Sin embargo, tampoco es una panacea educativa y se tiene que acotar su potencial en los ámbitos donde resulta pertinente y viable, como es el caso de la evaluación de algunas competencias genéricas o transversales y profesionales, a condición de que se privilegie su carácter retroalimentador, de comprensión y mejora en la formación de los profesionales.

La evaluación de competencias en educación superior no puede reducirse a la simple sustitución de instrumentos de lápiz y papel por pruebas de desempeño, no se trata de la aplicación de instrumentos "en frío", llámense rúbricas, bitácoras o portafolios. Se tiene que repensar la cultura misma de la evaluación y su abordaje ético, su sentido y función pedagógica y social. Implica un importante desarrollo metodológico y técnico, la formación en habilidades de co-auto y hetero evaluación. Conlleva un cambio en la lógica 
de la transposición didáctica para poder analizar y construir escenarios y situaciones de enseñanza-evaluación auténticas, y conduce a un sistema de operación y gestión de la evaluación que incluye la construcción y validación, puesta en práctica, seguimiento y realimentación de una diversidad de dispositivos, mediante la participación activa de los actores de la educación.

Se expusieron dos casos de evaluación auténtica de competencias en el contexto de la educación superior mexicana, con la intención de ilustrar distintas opciones de buena práctica, pero con la advertencia de que no son ejemplos paradigmáticos diseminados en todas las IES. Por el contrario, hemos encontrado que en la educación superior mexicana aún hay una gran distancia para lograr un cambio significativo en la cultura y los métodos de evaluación educativa, puesto que en las últimas décadas hemos atestiguado políticas contradictorias en materia de evaluación que han derivado en la desinformación o incluso rechazo entre las comunidades docentes (Velasco, Díaz Barriga y Tójar, 2017). El tema de la educación basada en competencias enfrenta diversas encrucijadas, pues se ha instrumentado sobre todo con base en visiones empresariales, estandarizantes, utilitarias y pensadas en tipos ideales de estudiantes y profesores. Hoy en día se requiere transitar a una visión de competencias pensadas desde la diversidad y la colectividad, no sólo centrada en los requerimientos para el desempeño de puestos en las empresas, sino con un mayor sentido social y con una mirada crítica de lo que se persigue respecto a fines y medios educativos. Al mismo tiempo, es importante entender la "no neutralidad" del enfoque, de hecho, de ningún enfoque educativo, y partir de la comprensión de las tensiones que genera todo cambio educativo, sobre todo, entre los contextos locales y globales, las características de los campos de conocimiento acotados y las situaciones problema de verdadera relevancia social.

Algunas conclusiones que deseamos puntualizar son las siguientes:

- La evaluación auténtica de competencias generales y profesionales en educación superior es relevante y útil cuando se enfoca a valorar sistémicamente procesos de construcción de conocimiento, solución de problemas o generación de respuestas creativas ante tareas complejas, no tiene como cometido evaluar la reproducción de cúmulos información declarativa o conceptual. Puede plantear estándares de desempeño, pero no es conveniente estandarizar comportamientos, menos aún fuera de contexto y mediante procedimientos exclusivos de heteroevaluación. Su mayor aporte reside en la reflexión sobre los aprendizajes, la comprensión situada de los mismos y el replanteamiento del currículo y de nuevos ciclos de enseñanzaaprendizaje encaminados a una mejor formación y práctica profesional con un sentido social, no sólo laboral.

- La autenticidad de la evaluación se refiere a que se plantean situaciones y escenarios de evaluación referidos a prácticas socioculturales que son importantes en el contexto del mundo real o en la esfera profesional en la que se desenvuelve la persona; de ninguna manera se restringen a "alinear" el mundo académico con las necesidades de las empresas.

- Consiste en una evaluación de desempeño que conlleva a poner el conocimiento en la acción en la medida en que enfrenta a la persona evaluada al reto de la integración y movilización de conocimientos, destrezas técnicas, estrategias, actitudes, etcétera. 
- Se interesa por explorar y modelar procesos y producciones de enseñanzaevaluación en contextos específicos, privilegiando las simulaciones situadas y los aprendizajes in situ, el trabajo por proyectos, el análisis de casos, las estaciones de competencia profesional, el aprendizaje servicio, entre otros.

- No se resuelve mediante la administración unilateral o única de un instrumento o técnica, consiste en un sistema donde convergen fuentes e instrumentos múltiples y situados y donde se promueve la participación del sujeto de la evaluación, al estudiante o profesional en formación, en procesos de toma de decisiones, autoevaluación y autorregulación de su propio aprendizaje y eventualmente del de sus pares.

- Las mejores experiencias de evaluación auténtica están acotadas al contexto situado de una comunidad profesional o en una institución educativa donde se participa en procesos autocríticos y cíclicos de teorización e investigación educativa que arroja evidencia empírica con la finalidad de perfeccionar el sistema y escuchar la voz de los implicados.

Reiteramos que la evaluación es una actividad social marcada por valores, no es independiente de la cultura o el contexto, y como antes se ha afirmado, influye directamente en lo que consideramos valioso, en lo que aprendemos y en cómo lo aprendemos. La evaluación puede limitar o promover determinados aprendizajes, así como condicionar su sentido, significado y funcionalidad. Esto es relevante para enfatizar que el tránsito hacia una aproximación competencial de corte sociocultural no puede quedarse en el asunto del tipo de instrumentos o técnicas a emplear, dado que la representación de la evaluación suele limitarse a una tarea netamente instrumental bajo el control de la autoridad. El hecho de trabajar con portafolios o emplear rúbricas no define en sí mismo que se ha arribado a un sistema de evaluación auténtica, menos aún que se ha adoptado la filosofía de la evaluación alternativa.

La filosofía subyacente al enfoque sociocultural de la evaluación auténtica enfrenta tensiones importantes con la evaluación imperante de la calidad, a gran escala y focalizada en la rendición de cuentas, que privilegia las pruebas nacionales e internacionales estandarizadas y con pretensiones de estar libres de sesgo cultural. En muchos escenarios los docentes se encuentran entre el desconocimiento y la encrucijada del tipo de enseñanza y evaluación a realizar, sobre todo cuando se les exige lograr determinados resultados en los exámenes de conocimiento, llegando incluso a condicionar su ingreso o situación laboral.

En el plano didáctico, es importante que los profesores comprendan que no hay evaluación auténtica cuando se emplean las rúbricas o los portafolios de aprendizaje simplemente con fines de evaluación sumativa o certificadora, cuando la toma de decisiones respecto al qué y cómo de la evaluación reside en el criterio unidireccional del docente (heteroevaluación) o se niega la posibilidad de autoevaluación del alumno o de los pares (co-evaluación) (Velasco, Díaz Barriga y Tójar, 2017).

Tanto la concepción de competencia como de su evaluación están en proceso de revisión crítica, y algunos autores avizoran nuevas perspectivas, aparejadas con demandas y escenarios educativos distintos. Finalmente, la comprensión de los procesos y ayudas pedagógicas que se relacionan con el aprendizaje de competencias, la controversia entre contenido y competencia, la pertinencia y viabilidad del enfoque en diversos campos 
disciplinares e interdisciplinares, el asunto de la transposición didáctica cuando se enseña por competencias, el diseño y gestión del currículo, entre otros, son temas sobre los que se requiere mayor teorización e investigación en el campo educativo. El tránsito hacia una noción de competencia como capacidad situada, donde se enfaticen modelos educativos que propugnen por la educación integral del ser humano, la inclusión y la justicia social, anticipan la posibilidad de recuperar una terminología propiamente educativa y social, no economicista o empresarial. En síntesis, junto al desarrollo de mejores sistemas e instrumentos de evaluación de competencias y aprendizajes complejos, habrá que trabajar en la transformación de la filosofía, teoría y práctica de la evaluación educativa en las universidades.

En todo caso, la noción de competencia tendrá un sentido educativo si logra superar la mirada tecnócrata de educación para satisfacer las demandas del mercado, atrapada en el discurso hegemónico del neoliberalismo, y logra transitar hacia la concreción de la educación del ser humano desde la aceptación de la diversidad, la inclusión y la equidad, reconociendo que el saber-hacer de las profesiones debe vincularse tanto a los contextos socioculturales como a una serie de compromisos ético-humanistas y de justicia social.

\section{Agradecimientos}

Agradecimiento al apoyo recibido de la DGAPA-UNAM mediante el proyecto PAPIME PE3002 17.

\section{Referencias}

Barrón, C. (1997). Perspectivas de la formación de profesionales para el siglo XXI. En A. de Alba (Coord.), El currículum universitario de cara al nuevo milenio (pp. 252-258). Ciudad de México: CESU- UNAM.

Barroso, R. y Siqueiros, M. (2015, noviembre). El diario interactivo en línea o e-diario como estrategia para desarrollar la reflexión durante la práctica en escenarios reales con estudiantes de la Licenciatura en Educación Primaria. Ponencia presentada en el XIII Congreso Nacional de Investigación Educativa, COMIE, Chihuahua, México.

Barroso, R. (2017, septiembre). El diario interactivo. Ponencia presentada en el Primer Congreso Internacional de Investigación Educativa y Formación Docente, DGESPE-SEP, Acapulco, México.

Cano, E, (2015). Evaluación por competencias en educación superior. Madrid: La Muralla.

Coll, C. (2007). Las competencias en la educación escolar: Algo más que una moda y mucho menos que un remedio. Aula de Innovación Educativa, 161, 34-39.

Darling-Hammond, L., Ancess, J. y Falk, B. (1995). Authentic assessment in action. Studies of schools and students at work. Nueva York, NY: Teachers College Press.

Del Rey, A. (2012). Las competencias en la escuela. Una vision crítica sobre el rendimiento escolar. Buenos Aires: Paidós.

Design-Based Research Collective. (2003). Design-based research: An emerging paradigm for educational inquiry. Educational Researcher, 32(1), 5-8.

Denyer, M., Furnémont, J., Poulain, R. y Vanloubbeeck, G. (2007). Las competencias en la educación. Un balance. Ciudad de México: Fondo de Cultura Económica. 
Díaz Barriga, A. (2006). El enfoque de competencias en la educación ¿Una alternativa o un disfraz de cambio? Perfiles Educativos, 28(111), 7-36.

Díaz Barriga, F. y Barrón, C. (2014). Curricular changes in higher Education in Mexico (20022012). Journal of Curriculum and Teaching, 3(2), 58-68.

Díaz Barriga, F. y Barroso, R. (2015). Los portafolios electrónicos como recurso de evaluación formativa y autorreflexión. En Guevara, G. et al. (Coords.), La evaluación docente en México (pp. 219-239). Ciudad de México: Fondo de Cultura Económica.

Espinosa, O. (2018). Evaluación de la competencia clínica en estudiantes de odontología mediante el examen clínico objetivo estructurado. Tesis doctoral. Universidad Nacional Autónoma de México, México.

Espinosa, O., Martínez, A., Sánchez, M. y Leenen, I. (2017). Análisis de un examen clínico objetivo estructurado en odontología desde la teoría de la generalizabilidad. Investigación en Educación Médica, 6(22), 109-118.

Fullan, M. (2001). The new meaning of educational change. Nueva York, NY: Teachers College Press.

Gibson, D. y Barrett, H. (2003). Directions in electronic portfolio development. Contemporary Issues in Technology and Teacher Education, 2(4), 559-576.

González, J. M., Wagenaar, R., y Beneitone, R. (2004). Tuning América Latina: un proyecto de las universidades. Revista Iberoamericana de Educación, 35, 151-164.

Jonnaert, P. (2002). Compétences et socio-constructivisme. Un cadre théorique, Bruselas: De BoeckUniversité.

López-Figueroa, M. (2014). El enfoque por competencias como factor de transformación educativa. Estudio de caso sobre concepciones y prácticas docentes en profesores de educación media superior. Tesis doctoral. Universidad Iberoamericana Puebla, México.

Loveless, A. y Williamson, B. (2017). Nuevas identidades de aprendizaje en la era digital. Madrid: Narcea.

Mulder, M., Weigel, T. y Collins, K. (2007). The concept of competence in the development of vocational education and training in selected EU member states: A critical analysis. Journal of Vocational Education and Training, 59(1), 67-88.

Perrenoud, Ph. (2004). Diez nuevas competencias para enseñar. Barcelona: Graó.

Pinar, W. (2003). International Handbook of Curriculum Research. Mahwah, Trenton, NJ: Lawrence Erlbaum.

Roe, R. (2003). ¿Qué hace competente a un psicólogo? Papeles del Psicólogo, 24(86), 1-12.

Romero, C. (2008). El cambio educativo: entre la inseguridad y la comunidad. Entrevista a Andy Hargreaves. Propuesta Educativa, 27(junio), 63-69.

Schön, D. (1992). La formación de profesionales reflexivos. Barcelona: Paidós.

Stobart, G. (2008). Tiempos de pruebas: Los usos y abusos de la evaluación. Madrid: Morata.

Velasco, L. C., Díaz Barriga, F. y Tójar, J. C. (2017). Acquisition and evaluation of competencies by the use of rubrics. Qualitative study on university faculty perceptions in Mexico. Procedia-Social and Behavioral Sciences, 237, 869-874. https://doi.org/10.1016/j.sbspro.2017.02.185.

Wenger, E. (2001). Comunidades de práctica. Aprendizaje, significado e identidad. Barcelona: Paidós. 


\section{Breve CV de la autora}

\section{Frida Díaz Barriga Arceo}

Doctora en Pedagogía y profesora titular C de tiempo completo de la Facultad de Psicología de la UNAM. Pertenece al Sistema Nacional de Investigadores, nivel 3. Coordina el Grupo de Investigación en Docencia, Diseño Educativo y TIC (GIDDET), reconocido como laboratorio de investigación por la Facultad de Psicología. Por invitación de la Organización de Estados Iberoamericanos (OEI) se integró en 2007 al Equipo de Expertos Iberoamericanos en Educación y TIC. Ha recibido las medallas Gabino Barreda, Sor Juana Inés de la Cruz (UNAM), el Premio Mexicano de Psicología 2009, la Medalla Anáhuac en Educación 2011 y el doctorado honoris causa por la UCI 2016. Tiene 227 publicaciones en revistas, libros de autor y coordinados, capítulos de libros y textos de divulgación. Ha dirigido más de 104 tesis donde los estudiantes se han graduado de licenciatura, maestría y doctorado. Asimismo, ha participado en una diversidad de congresos académicos y eventos de formación a nivel nacional e internacional; con periodicidad realiza estancias de investigación y formación a estudiantes de posgrado y docentes en universidades españolas y latinoamericanas. ORCID ID: 0000-0001-87201857.Email:diazfrida@prodigy.net.mx 